\title{
BMJ Prenatal alcohol exposure and Open childhood balance ability: findings from a UK birth cohort study
}

\author{
Rachel Humphriss, ${ }^{1,2}$ Amanda Hall, ${ }^{2,3}$ Margaret May, ${ }^{1}$ Luisa Zuccolo, ${ }^{1,4}$ \\ John Macleod ${ }^{1}$
}

To cite: Humphriss R, Hall A, May M, et al. Prenatal alcohol exposure and childhood balance ability: findings from a UK birth cohort study. BMJ Open 2013;3:e002718.

doi:10.1136/bmjopen-2013002718

- Prepublication history and additional material for this paper are available online. To view these files please visit the journal online (http://dx.doi.org/10.1136/ bmjopen-2013-002718).

Received 12 February 2013 Accepted 16 April 2013

This final article is available for use under the terms of the Creative Commons Attribution Non-Commercial 2.0 Licence; see http://bmjopen.bmj.com

For numbered affiliations see end of article.

\section{Correspondence to} Professor John Macleod; john.macleod@bristol.ac.uk

\section{ABSTRACT}

Objective: To investigate the association of prenatal alcohol exposure with balance in10-year-old children. Design: Population-based prospective longitudinal study.

Setting: Former Avon region of UK (Southwest England).

Participants: 6915 children from the Avon Longitudinal Study of Parents and Children who had a balance assessment at age 10 and had data on maternal alcohol consumption.

Outcome measures: 3 composite balance scores: dynamic balance (beam-walking), static balance eyes open, static balance eyes closed (heel-to-toe balance on a beam and standing on one leg, eyes open or closed).

Results: Most mothers (95.5\%) consumed no-tomoderate amounts (3-7 glasses/week) of alcohol during pregnancy. Higher total-alcohol consumption was associated with maternal-social advantage, whereas binge drinking ( $\geq 4$ units/day) and abstinence were associated with maternal social disadvantage. No evidence was found of an adverse effect of maternal-alcohol consumption on childhood balance. Higher maternal-alcohol use during pregnancy was generally associated with better offspring outcomes, with some specific effects appearing strong (static balance eyes open and moderate total alcohol exposure at 18 weeks, adjusted OR $1.23(95 \% \mathrm{Cl} 1.01$ to 1.49$)$; static balance eyes closed and moderate total alcohol exposure at 18 weeks, adjusted OR $1.25(95 \% \mathrm{Cl} 1.06$ to 1.48). Similar results were found for both paternal and postnatal maternal alcohol exposure. A Mendelianrandomization approach was used to estimate the association between maternal genotype and offspring balance using the non-synonymous variant rs $1229984^{\star} \mathrm{A}(A D H 1 B)$ to proxy for lower maternal alcohol consumption; no strong associations were found between this genotype/proxy and offspring balance.

Conclusions: No evidence was found to indicate that moderate maternal alcohol consumption in this population sample had an adverse effect on offspring balance at age 10. An apparent beneficial effect of higher total maternal alcohol consumption on offspring balance appeared likely to reflect residual confounding.

\section{ARTICLE SUMMARY}

Article focus

- Alcohol consumption during pregnancy has been found to have adverse effects on several neurodevelopmental outcomes. However, previous observational studies of the effect of prenatal alcohol exposure on childhood balance ability, an important neurodevelopmental outcome, have failed to reach consensus.

- This study investigates the association of prenatal alcohol exposure with balance in10-year-old children.

Key messages

- No evidence was found of an adverse effect of low-to-moderate maternal alcohol consumption on childhood balance. Rather, we found an apparently beneficial effect of prenatal alcohol exposure on balance at age 10 years.

- Analyses using maternal-paternal and timing of exposure comparisons, and using a Mendelian randomization approach (in which a genotype was used as a proxy for maternal alcohol consumption), suggested that these paradoxical findings were the result of residual confounding due to the association between higher alcohol use and social advantage in this population.

- It is therefore possible that apparently beneficial effects of low-to-moderate maternal alcohol consumption during pregnancy on offspring neurobehavioural outcomes reported by some other studies may be similarly non-causal.

Strengths and limitations of this study

- Using the Avon Longitudinal Study of Parents and Children data, we were able to investigate the possible effects of maternal alcohol use at levels typically seen in the general population on offspring balance in a large population-based sample of children, using prospective measures of both exposure and outcome and with additional measures allowing us to address the problem of confounding, in a way not previously possible.

- Limitations include the low test-retest reliability of our balance measures, a common problem with measures of childhood balance.

- Even with our relatively large sample size, it is likely that our genetic analyses were underpowered. 


\section{INTRODUCTION}

Balance is an important neurodevelopmental outcome in children, underpinning many motor skills. Balance problems in children can be associated with loss of confidence, low self-esteem and anxiety. ${ }^{2}$ Adverse effects of prenatal alcohol exposure on a variety of offspring neurodevelopmental outcomes have been well documented ${ }^{3-8}$ and animal models suggest that fetal alcohol exposure may adversely influence offspring balance. ${ }^{9}$ Animal models also suggest adverse effects of paternal alcohol use on a number of offspring outcomes; however, an adverse effect of paternal alcohol use on offspring balance has not been found. ${ }^{10}{ }^{11} \mathrm{~A}$ recent systematic review found no strong evidence of effects of prenatal maternal alcohol exposure at levels typically seen in the general population on offspring balance, but noted important limitations in the current evidence base including an absence of large general population-based prospective studies with adequate measures of both alcohol exposure and balance outcomes. ${ }^{12}$

In addition, observational studies of effects of prenatal maternal behavioural exposures on offspring outcomes are prone to residual confounding by factors related to social position, as maternal behaviour is often related to socioeconomic status (SES). Maternal alcohol use during pregnancy is patterned by SES: very high use, 'binge' drinking and complete abstinence from alcohol are associated with social disadvantage, while more moderate use is associated with social advantage. ${ }^{13}$ This, rather than any direct effect of alcohol consumption, may explain apparently beneficial associations between moderate maternal alcohol use during pregnancy and offspring neurocognitive outcomes. ${ }^{13} 14$

Different epidemiological approaches can be used to help identify causal effects of prenatal exposures including comparisons of maternal-paternal effects and of effects associated with different timings of exposure. ${ }^{15-17}$ Further, 'Mendelian randomization', which utilises genetic variation known to influence levels of the environmental exposure under examination, can be helpful. This approach is based on the assumption that since genotype is not usually associated with SES, it will not be associated with socially patterned confounding factors. ${ }^{18} 19$ Variants in alcohol dehydrogenase $(A D H)$ genes, particularly $A D H 1 B$, have previously shown robust associations with alcohol consumption. ${ }^{20}$ Specifically, a rare non-synonymous variant in $A D H 1 B$ has been shown to predispose to lower consumption before and during pregnancy. ${ }^{21}$ Maternal $A D H 1 B$ genotype can therefore be used as an instrument for maternal alcohol consumption (ie, in place of a self-reported measure of exposure) in analyses of effects of maternal alcohol use on offspring balance utilising the Mendelian randomization framework.

We used different epidemiological approaches to investigate the effects of prenatal alcohol exposure on the balance ability of 10-year-old children in a large general population sample.

\section{METHODS}

\section{Study population}

The study group was taken from the Avon Longitudinal Study of Parents and Children (ALSPAC) (http://www. bristol.ac.uk/alspac), a birth cohort of children born to pregnant women who were resident in the former Avon region of the UK and who were due to give birth between April 1991 and December 1992 (n=14 541 giving rise to 13988 live infants at 1 year). Full characteristics of mothers and children in the ALSPAC cohort have been described elsewhere. ${ }^{22}{ }^{23}$ Participants were informed in advance of all measures to be taken at the research clinic. Informed verbal consent was obtained and recorded at clinic attendance. Ethical approval for the study was obtained from the ALSPAC Law and Ethics Committee (IRB 00003312) and the Local Research Ethics Committees (Southmead, United Bristol Healthcare Trust, Frenchay and Weston Area Health Trust). Written consent was obtained for the use of genetic data (maternal genotype at rs1229984 in $A D H 1 B$ as described later).

The sample included all singleton-birth children from the original ALSPAC cohort who had undergone the 20 min balance assessment at age 10 (excluding children $(n=59)$ with significant physical disabilities and/or visual impairment, as identified by a teachers' questionnaire at age 7-8 years in the context of an assessment of special educational needs), and for whom data on maternal alcohol exposure at the time-points under consideration had been collected. Balance test results for up to 6915 children were available for analyses, depending on the completeness of the alcohol exposure data.

\section{Balance outcome measures}

Full details of the balance outcomes considered including details of their measurement and validity have been published elsewhere. ${ }^{24}$ In brief, at 10 years of age, these measures included: time to cross a $2 \mathrm{~m}$ balance beam, walking heel-to-toe; heel-to-toe balance on a beam, eyes open and closed (time balance maintained up to a maximum of $20 \mathrm{~s}$ ); and standing on one leg, eyes open and closed (time balance maintained up to a maximum of $20 \mathrm{~s}$ ). All children had two attempts at the beamwalking test and those who were unable to maintain their balance for the full $20 \mathrm{~s}$ had a second attempt at the tests of static balance. Composite scores were calculated as described elsewhere, ${ }^{24}$ resulting in three balance outcome measures: dynamic balance (DB); static balance eyes open (SBEO); and static balance eyes closed (SBEC). The DB and SBEC scores were both categorised into quartiles; the SBEO score was dichotomised into maximum score versus < maximum score as the distribution of this variable was heavily right skewed with more than half of children achieving the maximum score. The resulting categorical data were coded such that the first quartile of $\mathrm{DB} \quad(\mathrm{n}=1961,29.4 \%)$, the 'maximum score' of SBEO ( $\mathrm{n}=3751$ children, $56 \%)$ and 
the fourth quartile of SBEC $(\mathrm{n}=1618,24.5 \%)$ were all designated 'good balance'.

\section{Measures of alcohol exposure}

Maternal alcohol consumption at 18 weeks gestation and at 47 months postnatally was measured using self-report questionnaires. At 18 weeks gestation, mothers reported both their current consumption and their consumption prior to pregnancy. For each time point, the mothers reported the total number of glasses consumed per week categorised into none (0 glasses), low (1-2), moderate (3-7) and high (>7) consumption. The questionnaire defined a glass as, "a pub measure of spirits, half a pint of lager or cider, a wine glass of wine". At 18 weeks gestation, the mothers were also asked on how many days in the previous month they had drunk at least the equivalent of 4 units of alcohol, in order to examine the effects of binge drinking (categorised as never, 1-2, 3-4, $5-10,10+$ days and every day, which were recoded into none, 1-4, 5-10, 10+ days).

Paternal alcohol consumption was also measured by paternal self-report at 18 weeks gestation of the mother's pregnancy. Fathers' binge drinking was assessed as for mothers. In addition, the fathers were also asked about their total alcohol consumption during the first 3 months of pregnancy with response categories of never, less than 1 glass a week, at least 1 glass a week, at least 1 or 2 glasses every day, at least 3-9 glasses every day and at least 10 glasses every day. These categories were recoded into never, $<1 /$ week, $1+$ /week, $1+$ /day.

\section{Measures of potential confounders}

Potential confounding factors were considered based on evidence of their variation with maternal alcohol consumption and with offspring-balance outcomes. Measures of SES were recorded by maternal self-report questionnaires at approximately 8 weeks gestation. These were marital status (never married, previous marriage (widowed/divorced/separated), married); crowding index (number of people in the household divided by the number of rooms); home-ownership (mortgaged/ owned, rented/other); maternal occupational social class, classified in accordance with the British Registrar General's Classification (I professional, II intermediate, III-N skilled non-manual, III-M skilled manual, IV semiskilled manual/V unskilled manual); and ethnicity (white, non-white). Maternal parity (none, 1-2, 3+ previous children) and self-reported highest level of maternal education (CSE/vocational, O-levels, A-levels, degree) were recorded at 18 weeks gestation.

Maternal age was extracted from delivery data $(\leq 20$, $21-30,31-35$ and $>35$ years). The number of cigarettes smoked per day was recorded by the maternal self-report questionnaire at 18 weeks gestation. Mothers' smoking prepregnancy, during the first 3 months of pregnancy and at 18 weeks gestation were recorded (no smoking, $1-9,10-19,20+/$ day) along with maternal cannabis use (none vs any). Maternal caffeine consumption was recorded by self-report questionnaires at both 8 and 18 weeks gestation. The number of cups of caffeinated drinks (coffee, tea, cola) consumed per week were summed and then categorised into none; 1-21; 22-42; and $>42$.

Maternal depression was measured at 18 weeks gestation using the Edinburgh Postnatal Depression Scale, ${ }^{25}$ and categorised into not depressed $(<10)$ or possible depression $(\geq 10)$. Stressful life events during pregnancy were also measured at 18 weeks gestation giving a weighted-life-events score categorised into $0-1 ; 2-4 ; 5-7$; and $7+$.

\section{Genetic data}

Maternal DNA was extracted from peripheral blood collected as part of routine care during pregnancy ${ }^{22}$ as described previously. ${ }^{26}$ Single-nucleotide polymorphisms were genotyped by KBioscience (http://www. kbioscience.co.uk) using the KASPar chemistry, a competitive allele-specific PCR system using FRET quencher cassette oligos (http://www.kbioscience.co.uk/genotyping/ genotyping-chemistry.htm). Blind duplicates, plateidentifying repeat samples and Hardy-Weinberg equilibrium tests were used as quality control checks. Maternal genotype at rs1229984 in $A D H 1 B$ was available for a subset of 4335 mothers. ${ }^{21}$ The rare $\mathrm{A}$ allele of this variant has been found to be associated with lower alcohol consumption and less binge drinking during pregnancy. ${ }^{21}$

\section{Statistical analyses}

Analyses were conducted on a complete-case dataset, using STATA V.IC10 software. The $\chi^{2}$ test was used to compare our sample with the remaining (untested) cohort (table A1 web only). Ordered logistic regression was used to estimate associations between confounding variables and measures of both maternal and paternal alcohol exposure. Logistic and ordered logistic regression were used to estimate crude associations between the confounding variables and the binary balance outcome, SBEO, and the ordered categorical balance outcomes, DB and SBEC. Logistic and ordered logistic regression were also used to estimate both crude and adjusted associations (adjusting for confounders as listed in tables A1 and A2 web only) between the measures of maternal and paternal alcohol exposure and our balance outcome measures. Results are expressed as OR of better balance score with 95\% CI. Odds ratios (ORs) from models using maternal alcohol exposures were compared with those using paternal alcohol exposures.

For the Mendelian randomization analyses, given the rarity of the A allele (approximately 2\%) and the dominant mode of inheritance, AA and GA genotypes were combined, and are referred to as 'A allele carriers'. Data on $A D H 1 B$ genotype were available for 4257 of the white-European women, a total of $216(5.1 \%)$ who had the dominant rare A allele (2 homozygous, 214 heterozygous). 
Table 1 Alcohol consumption levels of women and their partners at each time point

\begin{tabular}{|c|c|c|c|}
\hline Alcohol exposure time-point & Measure & Categorisation & N (\%) \\
\hline \multirow[t]{4}{*}{ Maternal pre-pregnancy ${ }^{\star}$} & How often drunk alcohol & Never & $421(6.2)$ \\
\hline & & $<1$ glass/week & $2545(37.6)$ \\
\hline & & $\geq 1$ glass/week & $3006(44.5)$ \\
\hline & & $\geq 1$ glass/day & $788(11.7)$ \\
\hline \multirow[t]{4}{*}{ Maternal at 18 weeks gestation } & Total number of glasses/week & 0 & $4744(70.6)$ \\
\hline & & $1-2$ & 957 (14.2) \\
\hline & & $3-7$ & $718(10.7)$ \\
\hline & & $>7$ & $302(4.5)$ \\
\hline \multirow[t]{4}{*}{$\begin{array}{l}\text { Maternal binge drinking (at } 18 \text { weeks } \\
\text { gestation) }\end{array}$} & $\begin{array}{l}\text { Number of days in last month when } \geq 4 \text { glasses } \\
\text { drunk (binge drinking) }\end{array}$ & 0 & $5709(84.8)$ \\
\hline & & $1-4$ & $778(11.6)$ \\
\hline & & $5-10$ & $116(1.7)$ \\
\hline & & $>10$ & $129(1.9)$ \\
\hline \multirow[t]{4}{*}{ Maternal postnatal (47 months) } & Total number of glasses/week & 0 & $1756(28.7)$ \\
\hline & & $1-2$ & $1052(17.2)$ \\
\hline & & $3-7$ & $1918(31.4)$ \\
\hline & & $>7$ & $1387(22.7)$ \\
\hline \multirow[t]{4}{*}{ Paternal first 3 months pregnancy } & How often drunk alcohol & Never & 189 (3.5) \\
\hline & & $<1$ glass/week & $1200(22.3)$ \\
\hline & & $\geq 1$ glass/week & $2843(52.7)$ \\
\hline & & $\geq 1$ glass/day & $1158(21.5)$ \\
\hline \multirow[t]{4}{*}{$\begin{array}{l}\text { Paternal binge drinking (at } 18 \text { weeks } \\
\text { gestation) }\end{array}$} & $\begin{array}{l}\text { Number of days in last month when } \geq 4 \text { glasses } \\
\text { drunk }\end{array}$ & 0 & $901(16.6)$ \\
\hline & & $1-4$ & $2029(37.3)$ \\
\hline & & $5-10$ & $1449(26.6)$ \\
\hline & & $>10$ & 1063 (19.5) \\
\hline
\end{tabular}

Logistic regression was used to confirm that the rare $\mathrm{A}$ allele was associated with lower maternal alcohol consumption in this subset of ALSPAC mothers and to investigate possible associations between the confounding variables and genotype. Women of white-European origin only and their children were included to avoid population stratification, as the allele frequency of rs1229984 varies markedly across different populations, ${ }^{27}$ and patterns of alcohol drinking are culturally dependent. ${ }^{28}$ Logistic regression analyses were used to estimate the effect of carrying the A allele at this locus on the three balance outcomes.

\section{RESULTS}

\section{Sample characteristics}

The representativeness of the sample and the implications of this for external validity and the possible introduction of bias have been discussed previously. ${ }^{24} \mathrm{~A}$ comparison of the characteristics of the sample compared with the untested remainder of the cohort is given in table A1 web only.

Table 1 describes alcohol consumption levels of women and their partners at each measurement occasion. Of note is the low prevalence of high levels of maternal alcohol consumption during pregnancy.

Associations between the potential confounders and the measures of maternal alcohol exposure examined are given in table A2 web only. In general, higher total levels of maternal alcohol consumption were associated with higher SES and higher maternal age, whereas higher levels of binge drinking were associated with lower SES and lower maternal age. Non-white ethnicity was associated with lower levels of total alcohol consumption. Associations between the potential confounders and the three balance outcome measures are given in table A3 web only. In general, better static balance was associated with higher maternal education and higher social class.

\section{Associations between balance outcomes and maternal alcohol use}

Associations between the three balance outcome measures and maternal alcohol exposure are given in table 2. No strong evidence of an adverse effect of alcohol exposure on any of the outcomes was found. In general, higher total maternal-alcohol consumption during pregnancy was associated with better offspring balance, the strongest effects being seen in relation to static balance. These beneficial associations were found for SBEO and moderate total alcohol exposure at 18 weeks, adjusted OR 1.23 (1.01 to 1.49) and SBEC and moderate total alcohol exposure at 18 weeks, adjusted OR 1.25 (1.06 to 1.48 ). The direction of association between maternal binge drinking at 18 weeks and offspring balance outcomes was less consistent and 
Table 2 Associations between measures of alcohol consumption and balance outcomes

\begin{tabular}{|c|c|c|c|c|c|c|c|c|c|}
\hline \multirow[b]{2}{*}{ Alcohol exposure } & \multicolumn{3}{|c|}{ Dynamic balance } & \multicolumn{3}{|c|}{ Static balance eyes open } & \multicolumn{3}{|c|}{ Static balance eyes closed } \\
\hline & $\mathbf{N}$ & $\begin{array}{l}\begin{array}{l}\text { Crude OR } \\
(95 \% \mathrm{Cl})\end{array} \\
\end{array}$ & $\begin{array}{l}\text { Adjusted OR } \\
(95 \% \mathrm{Cl}) \\
\end{array}$ & $\mathbf{N}$ & $\begin{array}{l}\text { Crude OR } \\
(95 \% \mathrm{Cl}) \\
\end{array}$ & $\begin{array}{l}\text { Adjusted OR }{ }^{\star} \\
(95 \% \mathrm{Cl})\end{array}$ & $\mathbf{N}$ & $\begin{array}{l}\text { Crude OR } \\
(95 \% \mathrm{Cl}) \\
\end{array}$ & $\begin{array}{l}\text { Adjusted OR } \\
(95 \% \mathrm{Cl})\end{array}$ \\
\hline Maternal pre-pregnancy & 4646 & & & 4674 & & & 4621 & & \\
\hline Never & 237 & 1 (ref) & 1 (ref) & 238 & 1 (ref) & 1 (ref) & 236 & 1 (ref) & 1 (ref) \\
\hline$<1$ glass/week & 1692 & $1.08(0.85$ to 1.39$)$ & $1.10(0.86$ to 1.41$)$ & 1711 & $1.03(0.78$ to 1.35$)$ & $1.01(0.76$ to 1.33$)$ & 1686 & $1.04(0.82$ to 1.32$)$ & 0.99 (0.78 to 1.27$)$ \\
\hline$\geq 1$ glass/week & 2165 & 1.19 (0.93 to 1.51$)$ & $1.22(0.95$ to 1.56$)$ & 2170 & $1.10(0.84$ to 1.44$)$ & $1.04(0.79$ to 1.37$)$ & 2154 & $1.22(0.96$ to 1.54$)$ & 1.13 (0.89 to 1.44$)$ \\
\hline$\geq 1$ glass/day & 552 & 1.07 (0.82 to 1.42$)$ & $1.13(0.85$ to 1.50$)$ & 555 & 1.17 (0.86 to 1.59$)$ & 1.08 (0.79 to 1.49$)$ & 545 & 1.36 (1.04 to 1.78$)$ & $1.23(0.93$ to 1.63$)$ \\
\hline $\bar{p}$ Value & & 0.302 & 0.164 & & 0.119 & 0.453 & & $<0.001$ & 0.009 \\
\hline Maternal at 18 weeks & 4778 & & & 4806 & & & 4751 & & \\
\hline None & 3361 & 1 (ref) & 1 (ref) & 3379 & 1 (ref) & 1 (ref) & 3339 & 1 (ref) & 1 (ref) \\
\hline Low & 714 & $1.04(0.90$ to 1.20$)$ & 1.04 (0.90 to 1.20$)$ & 719 & 0.95 (0.81 to 1.12$)$ & 0.94 (0.79 to 1.10$)$ & 716 & 1.13 (0.98 to 1.31$)$ & $1.10(0.95$ to 1.27$)$ \\
\hline Moderate & 505 & $0.98(0.83$ to 1.15$)$ & $0.98(0.83$ to 1.15$)$ & 507 & 1.23 (1.01 to 1.47$)$ & 1.23 (1.01 to 1.49$)$ & 501 & 1.25 (1.06 to 1.48$)$ & 1.25 (1.06 to 1.48$)$ \\
\hline High & 198 & $1.04(0.81$ to 1.35$)$ & 1.07 (0.82 to 1.39$)$ & 201 & $1.05(0.79$ to 1.40$)$ & 1.06 (0.79 to 1.43$)$ & 195 & 1.07 (0.83 to 1.39$)$ & $1.14(0.88$ to 1.48$)$ \\
\hline p Value & & 0.878 & 0.797 & & 0.165 & 0.171 & & 0.014 & 0.009 \\
\hline Maternal binge drinking & 4776 & & & 4803 & & & 4748 & & \\
\hline None & 4106 & 1 (ref) & 1 (ref) & 4128 & 1 (ref) & 1 (ref) & 4084 & 1 (ref) & 1 (ref) \\
\hline $1-4$ days & 525 & 1.12 (0.96 to 1.32$)$ & 1.15 (0.97 to 1.35$)$ & 5299 & 0.97 (0.81 to 1.17$)$ & $1.02(0.84$ to 1.22$)$ & 518 & 0.95 (0.81 to 1.12$)$ & 1.04 (0.88 to 1.22$)$ \\
\hline $5-10$ days & 75 & 0.72 (0.49 to 1.06$)$ & 0.77 (0.52 to 1.15$)$ & 75 & 1.46 (0.91 to 2.37$)$ & 1.58 (0.97 to 2.58$)$ & 75 & 1.06 (0.70 to 1.60$)$ & $1.19(0.78$ to 1.81$)$ \\
\hline$>10$ days & 70 & 1.30 (0.82 to 2.05$)$ & 1.27 (0.80 to 2.00$)$ & 71 & $1.06(0.66$ to 1.71$)$ & 1.07 (0.66 to 1.73$)$ & 71 & 1.53 (1.00 to 2.35$)$ & 1.67 (1.09 to 2.58$)$ \\
\hline $\mathrm{p}$ Value & & 0.471 & 0.348 & & 0.493 & 0.288 & & 0.297 & 0.035 \\
\hline Maternal postnatal & 4369 & & & 4394 & & & 4340 & & \\
\hline None & 1187 & 1 (ref) & 1 (ref) & 1192 & 1 (ref) & 1 (ref) & 1178 & 1 (ref) & 1 (ref) \\
\hline Low & 741 & 0.93 (0.79 to 1.10$)$ & 0.93 (0.78 to 1.10$)$ & 746 & $1.12(0.93$ to 1.34$)$ & $1.10(0.92$ to 1.33$)$ & 735 & 1.08 (0.92 to 1.27$)$ & 1.03 (0.97 to 1.23$)$ \\
\hline Moderate & 1426 & 1.07 (0.93 to 1.23$)$ & 1.07 (0.93 to 1.24$)$ & 1433 & 1.07 (0.92 to 1.26$)$ & 1.04 (0.89 to 1.22$)$ & 1417 & 1.18 (1.03 to 1.36$)$ & 1.09 (0.95 to 1.26$)$ \\
\hline High & 1015 & 1.07 (0.92 to 1.24$)$ & 1.07 (0.92 to 1.26$)$ & 1023 & 1.12 (0.94 to 1.32$)$ & 1.07 (0.90 to 1.27$)$ & 1010 & 1.31 (1.12 to 1.52$)$ & 1.19 (1.02 to 1.39$)$ \\
\hline p Value & & 0.182 & 0.178 & & 0.252 & 0.576 & & $<0.001$ & 0.028 \\
\hline Paternal first 3 months & 3841 & & & 3865 & & & 3827 & & \\
\hline Never & 119 & 1 (ref) & 1 (ref) & 121 & 1 (ref) & 1 (ref) & 119 & 1 (ref) & 1 (ref) \\
\hline$<1$ glass/week & 812 & $0.86(0.60$ to 1.21$)$ & 0.84 (0.59 to 1.20$)$ & 816 & 1.67 (1.13 to 2.45$)$ & 1.62 (1.10 to 2.39$)$ & 809 & $1.22(0.86$ to 1.72$)$ & $1.12(0.78$ to 1.60$)$ \\
\hline$\geq 1$ glass/week & 2086 & 0.93 (0.67 to 1.31$)$ & 0.93 (0.66 to 1.31$)$ & 2097 & 1.65 (1.14 to 2.38$)$ & 1.54 (1.06 to 2.25$)$ & 2070 & 1.45 (1.04 to 2.02 ) & 1.29 (0.92 to 1.82$)$ \\
\hline$\geq 1$ glass/day & 824 & 0.97 (0.69 to 1.38$)$ & 0.97 (0.68 to 1.38$)$ & 831 & 1.79 (1.22 to 2.63$)$ & 1.65 (1.11 to 2.44$)$ & 829 & 1.58 (1.11 to 2.23 ) & 1.36 (0.95 to 1.94$)$ \\
\hline$\overline{\mathrm{p}}$ Value & & 0.286 & 0.286 & & 0.049 & 0.206 & & $<0.001$ & 0.013 \\
\hline Paternal binge drinking & 3958 & & & 3983 & & & 3942 & & \\
\hline None & 631 & 1 (ref) & 1 (ref) & 637 & 1 (ref) & 1 (ref) & 629 & 1 (ref) & 1 (ref) \\
\hline $1-4$ days & 1474 & $1.10(0.93$ to 1.30$)$ & $1.11(0.94$ to 1.31$)$ & 1481 & 1.15 (0.95 to 1.39$)$ & $1.15(0.95$ to 1.39$)$ & 1460 & $1.16(0.98$ to 1.38$)$ & $1.16(0.98$ to 1.37$)$ \\
\hline 5-10 days & 1083 & 1.11 (0.93 to 1.32$)$ & 1.13 (0.94 to 1.34$)$ & 1089 & 1.11 (0.92 to 1.36$)$ & 1.09 (0.89 to 1.33$)$ & 1082 & 1.16 (0.98 to 1.39$)$ & 1.13 (0.95 to 1.36$)$ \\
\hline$>10$ days & 770 & 1.10 (0.91 to 1.33$)$ & $1.13(0.93$ to 1.36$)$ & 776 & 1.12 (0.91 to 1.39$)$ & $1.10(0.89$ to 1.36$)$ & 771 & 1.21 (1.00 to 1.47$)$ & 1.20 (0.99 to 1.46$)$ \\
\hline p Value & & 0.394 & 0.268 & & 0.464 & 0.676 & & 0.086 & 0.132 \\
\hline
\end{tabular}

OR for 'good balance' by categories of alcohol exposure.

*Adjusted for confounders: marital status, crowding index, home ownership, parity, maternal education, ethnicity, maternal age, maternal social class, smoking, cannabis use, caffeine

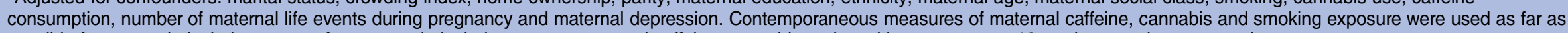
possible for prenatal alcohol exposure; for postnatal alcohol exposure, maternal caffeine, cannabis and smoking exposure at 18 weeks gestation was used. 
Table 3 Associations between alcohol exposure and ADH1B rs1229984

\begin{tabular}{|c|c|c|c|c|}
\hline Alcohol-exposure measure & Total $\mathbf{N}$ & $\begin{array}{l}\mathrm{N} \text { of } \mathrm{A} \text { allele } \\
\text { carriers }\end{array}$ & OR $(95 \% \mathrm{Cl})$ & p Value \\
\hline Total maternal alcohol consumption, prepregnancy & 4181 & 211 & $0.61(0.47$ to 0.79$)$ & $<0.001$ \\
\hline Total maternal alcohol consumption at 18 weeks gestation & 4171 & 210 & $0.66(0.48$ to 0.90$)$ & 0.010 \\
\hline Maternal binge drinking (at 18 weeks) & 4169 & 209 & $0.60(0.38$ to 0.96$)$ & 0.031 \\
\hline Total maternal alcohol consumption at 47 months postnatally & 3824 & 192 & $0.67(0.52$ to 0.87$)$ & 0.003 \\
\hline Total paternal alcohol consumption in first 3 months pregnancy & 3407 & 171 & $1.03(0.77$ to 1.37$)$ & 0.854 \\
\hline Paternal binge drinking & 3446 & 172 & $0.94(0.71$ to 1.24$)$ & 0.663 \\
\hline
\end{tabular}

effects were generally weak, though a higher level of binge drinking was associated with better SBEC in adjusted analyses, adjusted OR 1.67 (1.09 to 2.58).

\section{Maternal-paternal and timing of exposure comparisons}

Table 2 also gives the associations between paternal alcohol consumption during pregnancy and the balance outcomes and between maternal consumption after pregnancy and these balance outcome measures. In general, higher consumption is associated with better offspring balance though most associations are weak and imprecisely estimated. Maternal total alcohol consumption both before and after pregnancy and paternal total alcohol consumption at 18 weeks are all relatively strongly associated with better offspring SBEC. In addition, strong associations were found between paternal alcohol consumption in the first 3 months of pregnancy and better SBEO.

\section{Mendelian randomization analyses}

The maternal rs $1229984 * \mathrm{~A}$ allele $(A D H 1 B)$ was confirmed to be associated with lower levels of alcohol consumption in women before, during and after pregnancy, but not with paternal alcohol consumption (table 3), or with any of the confounding variables. There was no evidence that mothers genetically predisposed to lower alcohol consumption because of their $A D H 1 B$ genotype (A allele carriers) had offspring with poorer balance outcomes (table 4). All balance outcomes were better among offspring of mothers carrying the A allele (who would have been predisposed to lower alcohol consumption). However, this difference was small and very imprecisely estimated.

\section{DISCUSSION}

Self-reported higher total maternal alcohol use during pregnancy was generally associated with better offspring balance outcomes with some of these apparent effects being strong. A similar pattern of association was seen for maternal alcohol use before and after pregnancy and for paternal alcohol use during the mother's pregnancy. Maternal binge drinking during pregnancy showed a less consistent association with balance outcomes. Both paternal and maternal total alcohol use at any time point were higher among parents who were socially advantaged, although this association was not seen with maternal binge drinking. Statistical adjustment for a range of potential confounding factors including measures of social position generally made little difference to these estimates. Mothers whose genotype would have predisposed them to lower alcohol use at any time point had offspring with better balance outcomes though this apparent benefit was both small and very imprecisely estimated. Taken together, these results do not provide strong evidence of a specific effect (either adverse or beneficial) of maternal alcohol use during pregnancy. The beneficial effects apparent in several analyses, although theoretically plausible, ${ }^{29}$ were likely to have been the product of residual confounding.

\section{Comparison with existing evidence}

We are aware of no comparable evidence of effects of prenatal alcohol exposure on balance outcomes in school-age children. Our results are broadly consistent with those of most relevant studies in preschool children which have not found strong evidence of adverse effects. ${ }^{30-32}$ One previous longitudinal study reported

Table 4 Associations between $A D H 1 B$ rs1229984 and balance outcome measures, in subjects with white-European ethnicity

\begin{tabular}{|c|c|c|c|c|}
\hline Balance outcome measure & Total $\mathbf{N}$ & $\mathrm{N}$ of $\mathrm{A}$ allele carriers & OR $(95 \% \mathrm{Cl})$ & p Value \\
\hline Dynamic balance, age 10 & 4132 & 212 & 1.04 (0.81 to 1.33$)$ & 0.752 \\
\hline SBEO & 4148 & 213 & $1.12(0.85$ to 1.48$)$ & 0.425 \\
\hline SBEC & 4090 & 209 & $1.16(0.91$ to 1.49$)$ & 0.229 \\
\hline
\end{tabular}

OR for 'good balance' comparing A allele carriers to non-carriers.

$\mathrm{ADH}$, alcohol dehydrogenase; SBEC, static balance eyes closed; SBEO, static balance eyes open. 
adverse effects of maternal alcohol consumption prior to recognition of pregnancy on offspring balance at age 4 . However, this study over-sampled heavy drinkers and was limited in its ability to adjust for confounding. ${ }^{33}$ Some previous studies have found stronger evidence for adverse effects of low-to-moderate levels of maternal alcohol consumption during pregnancy and other neurobehavioural outcomes. ${ }^{34}{ }^{35}$ Conversely, other studies have suggested beneficial effects of such levels of maternal alcohol consumption on offspring neurocognitive $^{13}{ }^{14}$ and behavioural outcomes. ${ }^{29}$ Ostensibly, plausible biological mechanisms for these latter paradoxical benefits notwithstanding, our evidence suggests that they may in fact be non-causal and due to residual confounding. We also cannot exclude the possibility that residual confounding in our observational analyses may have masked a true adverse effect of maternal alcohol use. The unconfounded estimates we obtained using the 'Mendelian randomization' approach were compatible with an effect of lower maternal alcohol leading to better balance outcomes. However, if such an effect does exist, it appears to be small and in our analyses the probability of this finding arising by chance was high. We think the appropriate interpretation of our analyses is that they provide no strong evidence of an effect, either beneficial or detrimental, of moderate maternal alcohol use during pregnancy on offspring balance.

\section{Strengths and limitations}

Our main strengths were our ability to investigate the possible effects of maternal alcohol use at levels typically seen in the general population on offspring balance in a large population-based sample of children, using prospective measures of both exposure and outcome and with additional measures allowing us to address the problem of confounding, in a way not previously possible. The availability of paternally reported measures of paternal alcohol use is another strength, as many studies rely on maternal reports of paternal alcohol consumption.

Our study also had limitations. Our balance measures had low test-retest reliability, ${ }^{24}$ a common problem with measures of childhood balance. ${ }^{36-38}$ This may have led to random misclassification in our balance assessments and dilution of our estimates of effects of maternal alcohol use. Maternal alcohol use was self-reported which may have led to the introduction of bias. Since outcome measurement was independent of maternal self-report it is again likely that such bias would have led to dilution of apparent effects. Even with our relatively large sample size it is likely that our genetic analyses were underpowered. Absolute per allele differences in alcohol consumption would be small. Moreover, the approach depends on the 'intention to treat' principle. ${ }^{18}$ Larger sample sizes or meta-analysis of the results of several studies with relevant data may be required to answer this question. Rather than residual confounding, it is possible that apparently beneficial effects of alcohol consumption on some balance outcomes could have reflected chance findings arising from multiple comparisons. Had this been the case, however, we would have expected to see a mixture of beneficial and adverse effects which was not what we observed. Residual confounding appears an unlikely explanation for the association seen in the fully adjusted analysis between maternal binge drinking at 18 weeks and better static balance outcomes since maternal binge drinking was not associated with social advantage. Given the overall pattern of associations found, in particular, the lack of any consistent association between binge drinking and better balance and the results of the Mendelian randomization approach, we think a causal explanation for this finding is unlikely and suggest that it was probably due to chance.

There are issues of generalisability. The sample of children who underwent balance tests at age 10 were found to be socially advantaged compared with the remainder of the cohort (table A1 web only). ${ }^{24}$ This may have led to an under-representation of children exposed to more extreme patterns of alcohol use and thus have limited our opportunity to examine effects of these, although it is unlikely to have introduced important bias into the associations we describe. Selection bias due to missing data is also unlikely to affect the genetic analyses. ${ }^{39} \mathrm{We}$ examined effects of alcohol use patterns seen in women who were pregnant in the early 1990s. There is evidence that in UK women generally, both total alcohol consumption and levels of binge drinking have increased since the 1990s. ${ }^{40}$

\section{CONCLUSIONS}

We found no strong evidence for adverse effects of typical general population levels of maternal alcohol use during pregnancy on balance outcomes in school-age offspring. Results from analyses using the principle of 'Mendelian randomization' were compatible with such effects, but were underpowered. Paradoxical 'beneficial' effects found in some analyses were most likely a reflection of residual confounding by factors related to social position. Apparently beneficial effects of low-tomoderate maternal alcohol consumption during pregnancy on offspring-neurobehavioural outcomes reported in some other studies may be similarly non-causal.

\section{Author affiliations}

${ }^{1}$ School of Social and Community Medicine, University of Bristol, Bristol, UK ${ }^{2}$ Children's Hearing Centre, University Hospitals Bristol NHS Foundation Trust, Bristol, UK

${ }^{3}$ Centre for Hearing and Balance Studies, University of Bristol, Bristol, UK ${ }^{4}$ MRC Centre for Causal Analyses in Translational Epidemiology, School of Social and Community Medicine, University of Bristol, Bristol, UK

Acknowledgements We are extremely grateful to all the families who took part in this study, the midwives for their help in recruiting them and the whole ALSPAC team which includes interviewers, computer and laboratory technicians, clerical workers, research scientists, volunteers, managers, receptionists and nurses. The UK Medical Research Council, the Wellcome Trust and the University of Bristol provide core support for ALSPAC. The 
Alcohol Education and Research Council (AERC) provided a small grant in support of this project. The AERC had no role in the execution of this study. LZ was funded by an MRC Population Health Scientist fellowship (grant number G0902144). This publication is the work of all the authors and Rachel Humphriss and John Macleod will serve as guarantors for the contents of this paper.

Contributors RH performed the data analysis, led the drafting of the manuscript, provided expertise on balance and was a study guarantor. $\mathrm{AH}$ was involved in the methodology of the ALSPAC balance measures, participated in data collection and provided critical revision of the manuscript. MM provided consultation for data analysis methodology and strategy, interpretation of the results and critical revision of the manuscript. LZ provided consultation and expertise on genetic epidemiology methodology, interpretation of Mendelian randomization results and critical revision of the manuscript. JM provided epidemiological supervision, was involved in the strategy and interpretation of results, critical revision of the manuscript and was a study guarantor. All authors read and approved the final manuscript.

Funding Alcohol Education and Research Council (small grant). Competing interests None.

Ethics approval ALSPAC Law and Ethics Committee and the Local Research Ethics Committees.

Provenance and peer review Not commissioned; externally peer reviewed.

Data sharing statement For access to ALSPAC data please see: http://www. bristol.ac.uk/alspac/researchers/data-access

\section{REFERENCES}

1. Royal College of Physicians. Hearing and balance disorders achieving excellence in diagnosis and management. Report of a working party. London: RCP, 2008.

2. Bart O, Bar-Haim Y, Weizman E, et al. Balance treatment ameliorates anxiety and increases self-esteem in children with comorbid anxiety and balance disorder. Res Dev Disabil 2009;30:486-95.

3. Sampson PD, Sreissguth AP, Barr HM, et al. Neurobehavioural effects of prenatal alcohol: part II. Partial least squares analysis. Neurotoxicol Teratol 1989;11:477-91.

4. Coles CD, Brown RT, Smith IE, et al. Effects of prenatal alcohol exposure at school age. I. Physical and cognitive development. Neurotoxicol Teratol 1991;13:357-67.

5. Weinberg NZ. Cognitive and behavioural deficits associated with parental alcohol use. J Am Acad Child Adolesc Psychiatry 1997;36:1177-86.

6. Mattson SN, Goodman AM, Caine C, et al. Executive functioning in children with heavy prenatal alcohol exposure. Alcohol Clin Exp Res 1999;23:1808-15.

7. Kalberg WO, Provost B, Tollison SJ, et al. Comparison of motor delays in young children with fetal alcohol syndrome to those with prenatal alcohol exposure and with no prenatal alcohol exposure. Alcohol Clin Exp Res 2006;30:2037-45.

8. Williams Brown C, Carmichael Olson H, Croninger RG. Maternal alcohol consumption during pregnancy and infant social, mental and motor development. J Early Interv 2010;32:110-26.

9. Hannigan JH, Riley EP. Prenatal ethanol alters gait in rats. Alcohol 1989;5:451-4.

10. Abel EL. Paternal alcohol consumption affects grooming response in rat offspring. Alcohol 1991;8:21-3.

11. Abel EL. Paternal alcohol exposure and hyperactivity in rat offspring effects of amphetamine. Neurotoxicol Teratol 1993;15:445-9.

12. Humphriss RL, Hall A, Macleod J. Prenatal alcohol exposure and childhood balance: a systematic review. Paediatr Perinat Ep 2010;24:156-65.

13. Kelly Y, Sacker A, Gray R, et al. Light drinking during pregnancy: stil no increased risk for socioemotional difficulties or cognitive deficits at 5 years of age? J Epidemiol Commun H2012;66:41-8.

14. Kelly $Y$, Sacker A, Gray R, et al. Light drinking in pregnancy, a risk for behavioural problems and cognitive deficits at 3 years of age? Int $J$ Epidemiol 2009;38:129-40.

15. Davey Smith G. Assessing intrauterine influences on offspring health outcomes: can epidemiological studies yield robust findings? Basic Clin Pharmacol Toxicol 2008;102:245-56.
16. Alati R, Macleod J, Hickman M, et al. Intrauterine exposure to alcohol and tobacco use and childhood IQ: findings from a parental-offspring comparison within the Avon Longitudinal Study of Parents and Children (ALSPAC). Ped Res 2008;64:659-66.

17. Huizink AC. Moderate use of alcohol, tobacco and cannabis during pregnancy: new approaches and update on research findings. Reprod Toxicol 2009;28:143-51.

18. Davey Smith $\mathrm{G}$, Ebrahim S. What can mendelian randomisation tell us about modifiable behavioural and environmental exposures? $B M$ 2005;330:1076-9.

19. Lawlor DA, Harbord RM, Sterne JAC, et al. Mendelian randomisation: using genes as instruments for making causal inferences in epidemiology. Stat Med 2008;27:1133-63.

20. Macgregor S, Lind PA, Bucholz KK, et al. Associations of ADH and ALDH2 gene variation with self report alcohol reactions, consumption and dependence: an integrated analysis. Hum $\mathrm{Mol}$ Genet 2009;18:580-93.

21. Zuccolo L, Fitz-Simon N, Gray R, et al. A non-synonymous variant in $\mathrm{ADH} 1 \mathrm{~B}$ is strongly associated with prenatal alcohol use in a European sample of pregnant women. Hum Mol Genet 2009;18:4457-66.

22. Fraser A, Macdonald-Wallis C, Tilling K, et al. Cohort profile: the avon longitudinal study of parents and children: ALSPAC mothers cohort. Int J Epidemiol 2013;42:97-110.

23. Boyd A, Golding J, Macleod J, et al. Cohort profile: the 'children of the 90s'; the index offspring of the Avon Longitudinal Study of Parents and Children (ALSPAC). Int J Epidemiol 2013;42:111-27.

24. Humphriss R, Hall A, May M, et al. Balance ability of 7 and 10 year old children in the population: results from a large UK birth cohort study. Int J Ped Otorhinolaryngol 2011;75:106-13.

25. Cox JL, Holden JM, Sagovsky R. Detection of postnatal depression: development of the 10-item Edinburgh postnatal depression scale. Br J Psychiat 1987;150:782-6.

26. Jones RW, Ring S, Tynfield L, et al. A new human genetic resource: a DNA bank established as part of the Avon Longitudinal Study of Pregnancy and Childhood (ALSPAC). Eur J Hum Genet 2000;8:653-60.

27. Borinskaya S, Kal'ina N, Marusin A, et al. Distribution of the alcohol dehydrogenase $\mathrm{ADH}_{1 \mathrm{~B}}{ }^{*} 4 \mathrm{HH}$ is allele in Eurasia. Am J Hum Genet 2009;84:89-92.

28. World Health Organization (WHO). Global status report on Alcohol 2004, 2nd edn. Geneva, Switzerland: World Health Organization (WHO), 2004

29. Robinson M, Oddy $\mathrm{WH}$, McLean NJ, et al. Low-moderate prenatal alcohol exposure and risk to child behavioural development: a prospective cohort study. BJOG 2010;117:1139-52.

30. Jacobson JL, Jacobson SW, Sokol RJ, et al. Teratogenic effects of alcohol on infant development. Alcohol Clin Exp Res 1993;17:174-83.

31. Chandler LS, Richardson GA, Gallagher JD, et al. Prenatal exposure to alcohol and marijuana: effects on motor development of preschool children. Alcohol Clin Exp Res 1996;20:455-61.

32. Larroque B, Kaminski M, Dehaene $P$, et al. Prenatal alcohol exposure and signs of minor neurological dysfunction at preschoo age. Dev Med Child Neurol 2000;42:508-14.

33. Barr HM, Streissguth AP, Darby BL, et al. Prenatal exposure to alcohol, caffeine, tobacco and aspirin: effects on fine and gross motor performance in 4 year old children. Dev Psychol 1990;26:339-48.

34. Sood B, Delaney-Black V, Covington C, et al. Prenatal alcohol exposure and childhood behaviour at age 6 to 7 years: I. Dose-response effect. Pediatrics 2001;108:1-9.

35. Sayal K, Heron J, Golding J, et al. Prenatal alcohol exposure and gender differences in childhood mental health problems: a longitudinal population-based study. Pediatrics 2007;119:e426-34.

36. Brouwer B, Culham EG, Liston RAL, et al. Normal variability of postural measures: implications for the reliability of relative balance performance outcomes. Scand J Rehab Med 1998;30:131-7.

37. Liao H, Mao P, Hwang A. Test-retest reliability of balance tests in children with cerebral palsy. Dev Med Child Neurol 2001;43:180-6.

38. Geldhof E, Cardon G, De Bourdeaudhuij I, et al. Static and dynamic standing balance: test-retest reliability and reference values in 9 to 10 year old children. Eur J Pediatr 2006;165:779-86.

39. Davey Smith G, Lawlor DA, Harbord R, et al. Clustered environments and randomized genes: a fundamental distinction between conventional and genetic epidemiology. PLOS Med 2007;4: e352.

40. Smith L, Foxcroft D. Drinking in the UK. An exploration of trends Joseph Rowntree Foundation. 2009. http://www.safernorthdevon.co. uk/Publications/Publications\%20Drinking\%20in\%20UK-alcoholtrends-FULL\%5B1\%5D.pdf (accessed May 2011). 